\title{
BIAS COMPENSATED LEAST SQUARES \\ ESTIMATION OF CONTINUOUS TIME \\ OUTPUT ERROR MODELS IN THE CASE OF STOCHASTIC SAMPLING TIME JITTER
}

\author{
Frida Eng ${ }^{1}$ Fredrik Gustafsson ${ }^{2}$
}

\begin{abstract}
This work investigates how stochastic unmeasureable sampling jitter noise affects the result of system identification, and proposes a modification of known approaches to mitigate the effects of sampling jitter. By just assuming conventional additive measurement noise, the analysis shows that the identified model will get a bias in the transfer function amplitude that increases for higher frequencies. A frequency domain approach with a continuous time system model allows an analysis framework for sampling jitter noise. This leads to a bias compensated (weighted) least squares algorithm. A continuous time output error model is used for numerical illustration.
\end{abstract}

Keywords: system identification, stochastic systems, least-squares estimation, maximum likelihood, frequency domain

\section{INTRODUCTION}

Consider a deterministic signal model $s(t ; \theta)$, which may depend on an observed input. This work studies the problem of identifying the unknown parameter vector $\theta$, when the discrete time observations $y_{k}$ requested at time $t=k T$ ( $T$ denotes the sampling interval) are subject both to the usual additive measurement noise and also stochastic unmeasurable jitter noise $\tau_{k}$. That is, the observation includes the term $s\left(k T+\tau_{k} ; \theta\right)$, which becomes a stochastic variable. The case under consideration may occur when uniform sampling is requested, but the sensor for one or several reasons cannot measure exactly at that time instant, and the true sampling time is either unmeasurable, or the communication protocol does not allow to transport time stamps to each measurement.

As an illustration, consider the problem of measuring angular speeds. A standard sensor used in

\footnotetext{
1 Dept. of Elec. Eng., Linköping University, Sweden. Email: frida@isy.liu.se

2 Dept. of Elec. Eng., Linköping University, Sweden. Email: fredrik@isy.liu.se
}

for instance automotive anti-lock braking systems and engine control systems uses a cogged wheel fixed to an axle, where the time between each cog is measured by a timer. Sampling jitter occurs for two reasons mainly. The first is that the cogged wheel cannot be synchronized with the sampling clock, so the angular speed is computed for the last $\operatorname{cog}$ before the requested sampling time. The true sampling time is in principle known (though distributed over a cog duration), but communicating this would in many cases increase communication bandwidth and is not part of the protocol. Another more severe reason is road roughness and other sources causing axle vibrations that are superimposed in the angular domain, that cause a stochastic jitter in the time domain.

The general problem of nonuniform sampling is extensively treated in literature, see (Bilinskis and Mikelsons, 1992) and (Marvasti, 2001). In most publications, the sampling times are known, and the problem is to analyze leakage and alias effects. Another twist is to design sampling times to minimize aliasing. For stochastic sampling jitter, the distribution of $s\left(t+\tau_{k}\right)$ is derived in (Eng et 
al., 2005) and (Souders et al., 1990). These results will be used in this paper.

The basic approach is as follows. The frequency domain approach is to minimize the distance between the measurement and signal model Discrete Time Fourier Transforms (DTFT), $\| \operatorname{DTFT}\left(y_{k}\right)-$ $\operatorname{DTFT}\left(s\left(t+\tau_{k} ; \theta\right) \|\right.$ with respect to the parameters $\theta$ in some suitable norm. This frequency domain approach is standard in system identification (Pintelon and Schoukens, 2001; Ljung, 1999). A continuous time model for $s(t)$ is used to be able to analyze the sampling jitter effects. The analysis shows that by neglecting the jitter, the Fourier Transform (FT) of the signal model will suffer from an amplitude bias in $|\mathrm{FT}(s(t))|=$ $|S(f)|$ that increases for higher frequencies. Further, the larger jitter noise variance, the larger bias. The remedy is to compensate for the bias, and the closed form solution involves a frequency weighting window in the norm, $\| \operatorname{DTFT}\left(y_{k}\right)-$ $\int S(\varphi) w(f-\varphi) d \varphi \|$.

An inital study was presented in (Eng and Gustafsson, 2005), where the focus was on maximum likelihood estimation using both the first and second moments implied by the jitter sampling noise. Here, we concentrate on the first order moment and least squares estimate to gain further understanding for how the bias in the estimate lookl like, and to be able to move on to more realistic output error system identification experiments.

The outline is as follows. The system identification problem and main notation are presented in Section 2. In Section 3, the bias effect of sampling jitter noise on the frequency transform is derived, and the bias compensated least squares estimator is proposed. Section 4 illustrates the algorithm on a simulated numerical example. The work is concluded in Section 5.

\section{PROBLEM FORMULATION}

The general problem formulation looks as follows. The sensor is requested to sample uniformly, but delivers discrete time measurements corrupted by amplitude noise and sampling time jitter according to

$$
y_{k}=s\left(k T+\tau_{k} ; \theta\right)+v\left(k T+\tau_{k} ; \theta\right) .
$$

The signal, noise and jitter distributions are given by

$$
\begin{aligned}
s(t ; \theta) & =g_{\theta} * u(t), \\
v(t ; \theta) & =h_{\theta} * e(t), \\
\tau_{k} & \in p_{\theta}(\tau) .
\end{aligned}
$$

Here $u$ is a known input, $e$ is white noise with known characteristics, $g_{\theta}(t)$ denotes the system impulse response and $h_{\theta}(t)$ the noise dynamics. The jitter sampling noise is a sequence of independent stochastic variables with probability density function (pdf) $p_{\theta}(\tau)$. Both the signal, noise and sampling models can be parameterized in the unknown parameter vector $\theta$. We will primarily focus on continuous time systems, but discrete time systems where $g_{\theta}(t)$ and $h_{\theta}(t)$ are discrete time pulse responses are covered as well.

The system identification problems under consideration can be stated as estimating the parameter $\theta$ in a model structure

$$
\begin{aligned}
\mathcal{M}_{\mathcal{O E}} & : g_{\theta}(t), h_{\theta}(t)=\delta(t), p_{\theta}(\tau)=p(\tau), \\
\mathcal{M}_{\mathcal{B} \mathcal{J}} & : g_{\theta}(t), h_{\theta}(t), p_{\theta}(\tau)=p(\tau) \\
\mathcal{M}_{\mathcal{J O E}} & : g_{\theta}(t), h_{\theta}(t)=\delta(t), p_{\theta}(\tau), \\
\mathcal{M}_{\mathcal{J B J}}: & : g_{\theta}(t), h_{\theta}(t), p_{\theta}(\tau) .
\end{aligned}
$$

Here, OE denotes the output error and BJ the Box-Jenkins model structure, respectively, where the jitter distribution is known. JOE and JBJ are the corresponding problems where also the jitter noise distribution is parametrized.

Denote the Fourier transform of the measurements and signal model, respectively,

$$
\begin{aligned}
Y(f) & =\sum y_{k} e^{-i 2 \pi f k T}, \\
S(f ; \theta) & =G(f ; \theta) U(f),
\end{aligned}
$$

The general problem formulation is now to minimize the distance between the measurement and model in the frequency domain (Pintelon and Schoukens, 2001; Ljung, 1999),

$$
\hat{\theta}=\arg \min _{\theta}\|Y(f)-S(f ; \theta)\|,
$$

in some suitable norm, which may depend on $\theta$ implicitly via the noise dynamics and jitter distribution.

A main question at this stage is whether to express the measurement and signal in the Fourier Transform (FT), requiring continuous time signals, or in terms of the Discrete Time Fourier Transform (DTFT). Note that $Y(f)$ in (3) is the DTFT of the sequence $\left\{y_{k}\right\}$. Hereafter, the index $d$ on frequency functions denote the discrete time DTFT, while index $c$ denotes continuous time FT. Hence, a first attempt would be to either use $\left\|Y_{d}(f)-S_{d}(f ; \theta)\right\|$ or $\left\|Y_{c}(f)-S_{c}(f ; \theta)\right\|$ in $(5)$. However, neither one is attractive:

- A wellknown property of the Fourier transform is that

$$
Y_{d}(f)=\int Y_{c}(\psi) \Gamma_{N}^{p}(f-\psi) \mathrm{d} \psi,
$$

where the so called Dirichlet function is defined as

$$
\Gamma_{N}^{p}(f)=e^{-i \pi f(N-1) T} \frac{\sin (\pi f N T)}{\sin (\pi f T)} .
$$

The Dirichlet function is shown in Fig. 1, and its local behaviour describes the effects 
of leakage and its $1 / T$ periodicity describes aliasing. The problem is that there is no unambitious way to solve $(6)$ for $Y_{c}(f)$. That is, $Y_{d}(f)$ has to be used.

- There is no known way how to analyze sampling jitter effects in $S_{d}(f ; \theta)$. On the other hand, the jitter effects on a continuous time signal is described in the literature, see for instance (Eng et al., 2005) and (Souders et al., 1990). Hence, a continuous time model should be used.

The solution is to apply the leakage and aliasing window to the continuous time model. That is, the general system identification approach advocated here can be written as

$$
\hat{\theta}=\arg \min _{\theta}\left\|Y_{d}(f)-\int S_{c}(\psi ; \theta) \Gamma_{N}^{p}(f-\psi) \mathrm{d} \psi\right\| .
$$

This problem is closely related to the approach (Gillberg and Ljung, 2005) for the special case of no jitter noise, and (Gillberg and Gustafsson, 2005) for the case of non-uniform sampling (but deterministic known sampling times). Note also that the leakage can also be interpreted as a originating from a transient in the signal model caused by an unknown initial state. An alternative dual approach is based on estimating this initial states as studied in (Schoukens et al., 2005; Pintelon and Schoukens, 2001).

The purpose of this work is to analyze the bias contribution from jitter sampling noise on the signal model $S_{c}(f ; \theta)$, and modify the criterion (8) accordingly.

\section{BIAS COMPENSATION}

Using the least squares (LS) norm in (8) over a discrete set of frequencies yields

$\hat{\theta}^{L S}=\arg \min _{\theta} \sum_{f}\left|Y_{d}(f)-\int S_{c}(\psi ; \theta) \Gamma_{N}^{p}(f-\psi) \mathrm{d} \psi\right|^{2}$.

Similarly to (Pintelon and Schoukens, 2005) and (Gillberg and Gustafsson, 2005), the frequency set under consideration is arbitrary, in order to have the freedom to highlight certain frequency regions of interest. First, the bias in $Y_{d}(f)$ due to sampling jitter is derived. Then, the bias compensated LS (BCLS) estimate is defined. In the following two subsections, the dependence on the parameter $\theta$ will be implicit.

\subsection{Bias in $Y_{d}(f)$}

The DTFT (3) of the sequence $y_{k}$ using the FT $S(f)=\int s(t) e^{-i 2 \pi f t} d t$ and the sampling jitter model (1) can be written

$$
\begin{aligned}
Y_{d}(f) & =\sum_{k=0}^{N-1} y_{k} e^{-i 2 \pi f k T} \\
& =\sum_{k=0}^{N-1}\left(s\left(k T+\tau_{k}\right)+v_{k}\right) e^{-i 2 \pi f k T} \\
& =\hat{S}_{d}(f)+\underbrace{\sum_{k=0}^{N-1} v_{k} e^{-i 2 \pi f k T}}_{\hat{V}(f)} .
\end{aligned}
$$

Using the inverse FT $s(t)=\frac{1}{2 \pi} \int S_{c}(f) e^{i 2 \pi f t} d t$, the sampling noise part becomes

$$
\begin{aligned}
\hat{S}_{d}(f) & =\sum_{k=0}^{N} s\left(k T+\tau_{k}\right) e^{-i 2 \pi f k T} \\
& =\sum_{k=0}^{N-1} \frac{1}{2 \pi} \int S_{c}(\varphi) e^{i 2 \pi \varphi\left(k T+\tau_{k}\right)} \mathrm{d} \varphi e^{-i 2 \pi f k T} \\
& =\frac{1}{2 \pi} \int S_{c}(\varphi) W(f, \varphi) \mathrm{d} \varphi,
\end{aligned}
$$

where

$$
W(f, \varphi) \triangleq \sum_{k=0}^{N-1} e^{i 2 \pi(\varphi-f) k T} e^{i 2 \pi \varphi \tau_{k}} .
$$

The term $W$ is a stochastic frequency window, completely describing the jitter, leakage and aliasing effects in the signal term $S_{d}(f)$ of $Y_{d}(f)$. Note that $W(f, \varphi)=\Gamma_{N}^{p}(f-\varphi)$ in the case of no jitter, $\tau_{k}=0$. It should be stressed that this continuous time frequency domain approach is perhaps the only way to explicitly separate the signal amplitude noise and sampling jitter noise.

\subsection{First moment of $W$}

The moments of the transform $Y_{d}(f)$ have an explicit dependence on the signal transform $S_{c}(f)$. 2 The mean value of $Y_{d}(f)$ in $(3)$ is

$$
\begin{aligned}
\mu_{Y}(f) & =\mathrm{E}\left[Y_{d}(f)\right]=\mathrm{E}\left[\hat{S}_{d}(f)\right] \\
& =\frac{1}{2 \pi} \int S_{c}(\varphi) \mathrm{E}[W(f, \varphi)] \mathrm{d} \varphi \\
& =\frac{1}{2 \pi} \int S_{c}(\varphi) \mu_{W}(f, \varphi) \mathrm{d} \varphi
\end{aligned}
$$

and $\mu_{W}=E[W]$ is given by the following lemma.

Lemma 1. (Mean value). The mean value, $\mu_{W}$, with respect to $\tau_{k}$, of the stochastic window, $W(f, \varphi)$, defined in Eq. (12), is

$$
\mu_{W}(f, \varphi)=\gamma^{g}(-\varphi) \Gamma_{N}^{p}(f-\varphi),
$$

where $\gamma^{g}(f)$ is the characteristic function $\gamma^{g}(f)=$ $E\left[e^{-i 2 \pi f \tau}\right]$ of $\tau$ and $\Gamma_{N}^{p}(f)$ is the Dirichlet function (7).

\section{PROOF.}




$$
\begin{aligned}
\mu_{W}(f, \varphi) & =\mathrm{E}[W(f, \varphi)] \\
& =\sum_{k=0}^{N-1} e^{i 2 \pi(\varphi-f) k T} \mathrm{E}\left[e^{i 2 \pi \varphi \tau_{k}}\right] \\
& =\gamma^{g}(-\varphi) \underbrace{\frac{1-e^{i 2 \pi(\varphi-f) N T}}{1-e^{i 2 \pi(\varphi-f) T}}}_{\Gamma_{N}^{p}(f-\varphi)},
\end{aligned}
$$

where we recognize the characteristic function, $\gamma^{g}(f)=E\left[e^{-i 2 \pi f \tau}\right]$ and the Dirichlet function, $\Gamma_{N}^{p}$.

The characteristic function $\gamma^{g}(f)=\gamma^{g}(f ; \theta)$ models damping corresponding to the sampling jitter noise. It will include possible distribution parameters from $p_{\theta}(\tau)$ in $(2)$. Its properties include:

- $\left|\gamma^{g}(g)\right| \leq 1, \forall f$.

- $\gamma^{g}(f)=1$ iff there is no jitter noise, so $\tau_{k}=0$, $\forall k$.

- $\left|\gamma^{g}(f)\right| \rightarrow 0$, when $f \rightarrow \infty$.

- The decay rate of $\left|\gamma^{g}(f)\right|$ is faster the larger jitter noise variance.

The second property implies that $\mu_{W}(f, \varphi)=$ $W(f, \varphi)=\Gamma_{N}^{p}(f-\varphi)$ for the case of no jitter noise.

\subsection{Bias Compensated Least Squares Estimate}

With the complete knowledge of both the leakage and jitter effect on the measurement DTFT we can include this in the parameter estimation as

$$
\hat{\theta}^{B C L S}=\arg \min _{\theta} \sum_{f}\left|Y_{d}(f)-\mu_{Y}(f ; \theta)\right|^{2}
$$

where

$$
\mu_{Y}(f ; \theta)=\frac{1}{2 \pi} \int S_{c}(\psi ; \theta) \gamma^{g}(-\psi ; \theta) \Gamma_{N}^{p}(f-\psi) \mathrm{d} \psi .
$$

Both the signal $S_{c}(f ; \theta)$ and jitter pdf $p(\tau ; \theta)$, and thus characteristic function $\gamma^{g}(f ; \theta)$, may depend on the unknown parameter vector $\theta$.

In Fig. 1 the periodic Dirichlet function $\Gamma_{N}^{p}$ is shown together with the characteristic function $\gamma^{g}$, for a rectangular and Gaussian distribution. The jitter effect is shown as a damping of high frequencies, and excluding this effect will give biased estimates. The mean value of $Y$ will still be periodic, but alias effects are reduced from the scaling with $\gamma^{g}$.

\subsection{Illustration}

Consider a sinusoidal signal with one frequency $f_{0}$, $s(t)=\sin \left(2 \pi f_{0} t\right)$. Note that $S_{d}(f)=S_{d}(f+1 / T)$, so frequency shifts as multiples of the sampling interval will not affect the signal model as seen after

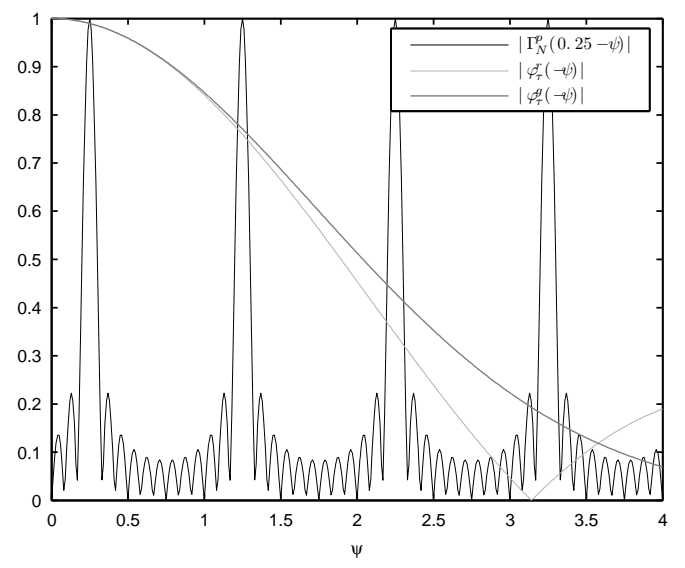

Fig. 1. Example of the periodic Dirichlet function, $\Gamma_{N}^{p}(0.25-\psi)$ (black), and the characteristic function, $\gamma^{g}(-\psi)$, for a rectangular (r, light gray) and a Gaussian (g, thicker medium gray) distribution with the same variance.

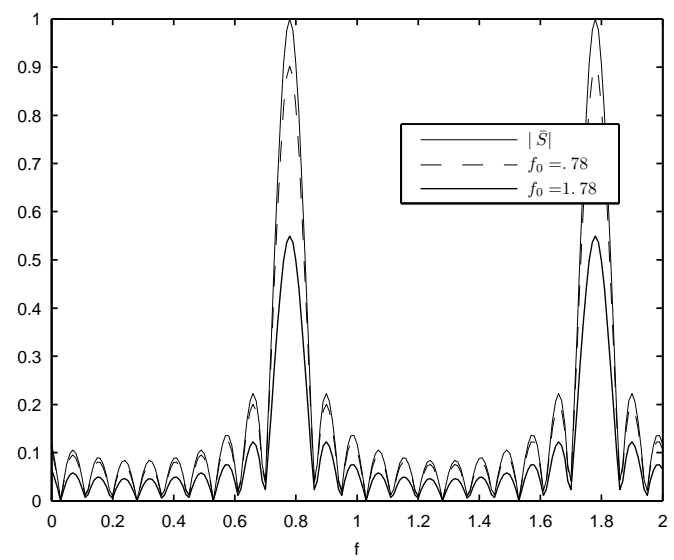

Fig. 2. The difference between $\mid S_{d}(f)$ and $\left|\mathrm{E}\left[Y_{d}(f)\right]\right|$, for two cases of single-frequencysignals.

convolution with the leakage and alias window. However, the observations are subject to jitter, and we now have a tool to describe the damping (bias) on $Y_{d}(f)$. We see directly from (14) that the damping is given by the characteristic function $\gamma^{g}\left(-f_{0}\right)$. The difference between $S_{d}(f)$ and $E\left[Y_{d}(f)\right]$ is shown in Fig. 2, for the two cases with $f_{0}=0.78$ and $f_{0}=1.78$. From Fig. 1 we know that the difference in amplitude is larger for higher frequencies $f_{0}$. This is one reason why we can find frequencies above $f_{N}=1 / T$, since a signal with frequency $f_{0}$ will be less damped than a signal with frequency $f_{0}+1 / T$.

\section{EXAMPLES}

A proof of concept example was presented in (Eng and Gustafsson, 2005) using a pure sinusoid, where the amplitude, frequency and jitter distribution were simultaneously identified. Here, continuous time output error models are considered. 
Table 1 . Mean value and standard deviation of $\hat{\theta}=[\hat{n} \hat{a}]$ and $\hat{\sigma}=\hat{a} / \sqrt{3(\hat{n}+1)}$ for the pdf (15). Gaussian noise is used in last two cases.

\begin{tabular}{|c|ccc||ccc|}
\hline Model $(15)$ & $n$ & $a$ & $\sigma$ & $n$ & $a$ & $\sigma$ \\
\hline \hline$\theta^{o}$ & 0 & 0.5 & $1 / \sqrt{12} \approx 0.2887$ & 0 & $1 / \sqrt{12} \approx 0.2887$ & $1 / 6 \approx 0.1667$ \\
\hline $\mathrm{E}\left[\hat{\theta}^{B C L S}\right]$ & 0.2145 & 0.4683 & 0.2599 & 1.1818 & 0.3759 & 0.1682 \\
$\operatorname{Std}\left[\hat{\theta}^{B C L S}\right]$ & 0.7360 & 0.0678 & 0.0489 & 1.6815 & 0.1186 & 0.0649 \\
\hline$\theta^{o}$ & 2 & 0.5 & $1 / 6 \approx 0.1667$ & 2 & $1 / \sqrt{12} \approx 0.2887$ & $1 /(6 \sqrt{3}) \approx 0.0962$ \\
\hline $\mathrm{E}\left[\hat{\theta}^{B C L S}\right]$ & 1.1386 & 0.3752 & 0.1676 & 1.6816 & 0.2618 & 0.1018 \\
$\operatorname{Std}\left[\hat{\theta}^{B C L S}\right]$ & 1.6608 & 0.1088 & 0.0592 & 1.9972 & 0.1553 & 0.0607 \\
\hline$\theta^{o}$ & $\rightarrow \infty$ & $\sigma^{o} \sqrt{3\left(n^{o}+1\right)}$ & $1 / 6 \approx 0.1667$ & $\rightarrow \infty$ & $\sigma^{o} \sqrt{3\left(n^{o}+1\right)}$ & $1 /(6 \sqrt{3}) \approx 0.0962$ \\
\hline $\mathrm{E}\left[\hat{\theta}^{B C L S}\right]$ & 1.8175 & 0.3267 & 0.1281 & 1.8807 & 0.1958 & 0.0752 \\
$\operatorname{Std}\left[\hat{\theta}^{B C L S}\right]$ & 2.0357 & 0.1607 & 0.0678 & 2.1966 & 0.1425 & 0.0530 \\
\hline
\end{tabular}

4.1 Known first order OE model with unknown jitter pdf

Here, we focus on finding the distribution of the sampling noise. We choose a parameterization based on a sum of $n$ uniformly distributed variables in the interval $[-a / n, a / n]$. That is, the support of the pdf $p_{\theta}(\tau)$ is always $[-a, a]$, and its parameters are $\theta=[n, a]$. The characteristic function of $p_{\theta}(\tau)$ can be written as (a sum of stochastic variables corresponds to convolving their pdf's, which in turns corresponds to multiplying their characteristic functions)

$$
\gamma_{\theta}^{g}(f)=\left(\frac{\sin \left(\frac{\pi f a}{n+1}\right)}{\frac{\pi f a}{n+1}}\right)^{n+1}=\operatorname{sinc}\left(\frac{f a}{n+1}\right)^{n+1} .
$$

The expected value is 0 and the variance is $\frac{1}{n+1} \frac{a^{2}}{3}$. Setting $n=0$ yields a rectangular distribution, $n=1$ a triangular distribution, and increasing $n$ makes the distribution converging to a normal distribution (with the same variance). Thus, a higher $n$ indicates a more narrow distribution. See Fig. 3 for a few examples of the pdf and corresponding (of equal variance) Gaussian distribution.

For this example we chose

$$
\mathcal{M}: g_{\theta}(t)=g(t), h_{\theta}(t)=\delta(t), p_{\theta}(\tau),
$$

with $g(t)=\mathcal{L}^{-1}\left(\frac{1}{s+2}\right)$.

We use $p_{\theta}(\tau)$ given by the CF in Eq. (15), for $[n, a]=[0, T / 2],[0, T / 2 \sqrt{3}],[2, T / 2]$, and $[2, T / 2 \sqrt{3}]$. We also let the true pdf be a zeromean Gaussian distribution with the same variance as the two last cases above, namely $\sigma^{2}=$ $(T / 6)^{2}$ and $\sigma^{2}=(T / 6 \sqrt{3})^{2}$. The input $u(t)$ is a Pseudo Random Binary Shift (PRBS) signal, which jumps between 0 and 1 at time intervals of exponentially distributed lengths, with a mean value of $8 \mathrm{~s}$. The measurement noise $v(t)$ is zeromean Gaussian with standard deviation 0.01. In Fig. 4, an example of the input $u(t)$ and output $y(t)$ is given for the above settings.

For the six different pdf's, we evaluate the mean value and the standard deviation of the estimated parameters, $\theta$, based on 128 different realizations

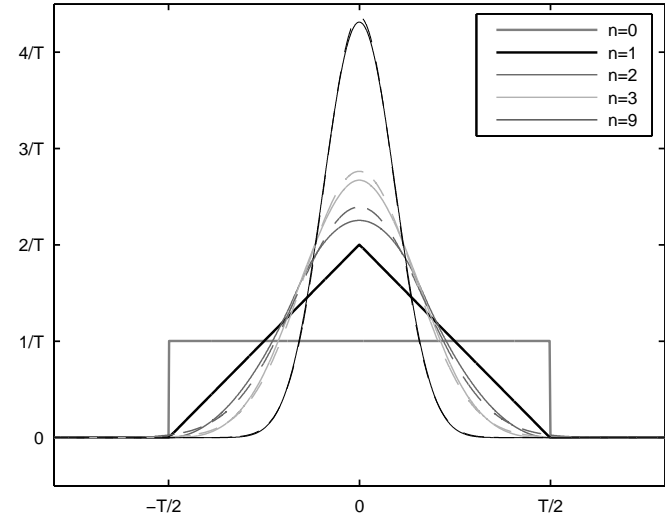

Fig. 3. The solid lines show the pdf, $p_{\theta}(\tau)$, corresponding to different values of $\theta$, when the CF is $\gamma^{g}(f)=\operatorname{sinc}\left(\frac{f a}{n+1}\right)^{n+1}$. For the higher values of $n$, the dashed lines show the Gaussian bells with the same variance as the parameterized pdf. The difference is almost invisible in this plot for $n=9$.

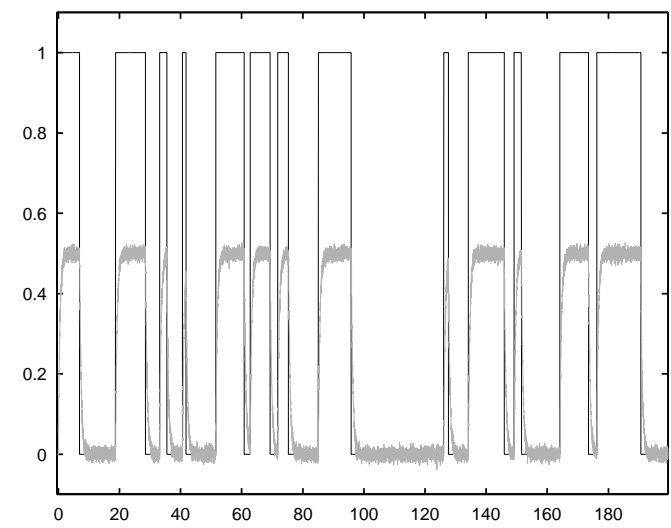

Fig. 4. Example of a PRBS signal $(u(t)$ black $)$ and the output from $G(s)=1 /(s+2),(y(t)$ gray $)$, in continuous time.

of the sampling noise sequence and the input. The result is shown in Table 1 , for both the ML and LS estimate. We also study the estimate of the standard deviation $\sigma$, given by $\hat{n}$ and $\hat{a}$. We can conclude the following:

- It seems quite easy to find the correct standard deviation $\sigma$ for the sampling noise, while the exact parameter constellation, $\left[\begin{array}{ll}n & a\end{array}\right]$, is 
harder. This can be due to too few samples $(N=200)$ to be able to estimate the shape of the distribution.

- The distribution with $n=0, a=0.5$ has best results.

- The ML estimate has the smallest error for all cases.

- True pdf's outside the model class pose no problem to the estimation of $\sigma$.

4.2 Unknown second order OE model with known jitter pdf

Table 2. Mean value and standard deviation of $\hat{\theta}=\left[\begin{array}{lll}\hat{k}_{0} & \hat{z} & \hat{p}_{r} \\ p_{i}\end{array}\right]^{T}$ in the system

(17).

\begin{tabular}{|c|cccc|}
\hline Model $(17)$ & $k_{0}$ & $z$ & $p_{r}$ & $p_{j}$ \\
\hline$\theta^{\circ}$ & 0.2 & 0.5 & -0.25 & 0.3 \\
\hline $\mathrm{E}\left[\hat{\theta}^{B C L S}\right]$ & 0.2000 & 0.5012 & -0.2504 & 0.3001 \\
$\operatorname{Std}\left[\hat{\theta}^{B C L S}\right]$ & 0.0025 & 0.0135 & 0.0019 & 0.0029 \\
\hline
\end{tabular}

Now, let us focus on the linear system, $g(t)$. We show the results for a second order system, specified by its transfer function $G$. The model is

$$
\mathcal{M}: g_{\theta}(t), h_{\theta}(t)=\delta(t), p_{\theta}(\tau)=p(\tau) .
$$

The system $g(t)=\mathcal{L}^{-1}(G(s))$ is parameterized as

$$
\begin{aligned}
G_{\theta}(s) & =k_{0} \frac{s+z}{\left(s-\left(p_{r}+i p_{i}\right)\right)\left(s-\left(p_{r}-i p_{i}\right)\right)} \\
\theta & =\left[\begin{array}{llll}
k_{0} & z & p_{r} & p_{i}
\end{array}\right]^{T}
\end{aligned}
$$

with the true parameters being $\theta^{0}=\left[\begin{array}{llll}0.2 & 0.5 & -0.25 & 0.3\end{array}\right]^{T}$ during this run. The sampling noise distribution, $p(\tau)$, is known and given by Eq. (15) with $n=2$ and $a=T / 2 / \sqrt{3} \approx 0.2887$. The input $u(t)$ is a Pseudo Random Binary Shift (PRBS) signal, which jumps between 0 and 1 at time intervals of exponentially distributed lengths, with a mean value of $8 \mathrm{~s}$. The measurement noise $v(t)$ is zeromean Gaussian with standard deviation 0.01.

The result for the ML and LS estimation is shown in Table 2, and it is clear that both methods give unbiased estimates with small variances. It is as easy to estimate four parameters of a linear system as it was finding the amplitude and frequency for a single sinusoid.

\section{CONCLUSIONS}

The stochastic jitter noise in the model $y_{k}=$ $s\left(k T+\tau_{k} ; \theta\right)+v_{k}$ should not be neglected and included in the measurement noise $v_{k}$. The bias compensated least squares estimator describes both the effects of jitter and the remedy:

$$
\begin{aligned}
\hat{\theta}^{B C L S} & =\arg \min _{\theta} \sum_{f} \mid Y(f)- \\
& \left.\frac{1}{2 \pi} \int S(\psi ; \theta) \gamma^{g}(-\psi ; \theta) \Gamma_{N}^{p}(f-\psi) \mathrm{d} \psi\right|^{2} .
\end{aligned}
$$

This criteria minimizes the LS distance between measurement TDFT and the model's FT, taking leakage and alias effects into account via a convolution with the Dirichlet window $\Gamma_{N}^{p}(f-\psi)$. The characteristic function $\gamma^{g}(-\psi ; \theta)$ of the jitter noise implies a scaling of the signal model $S_{c}(\phi)$. By neglecting this, a bias in $S_{c}(f)$ would result.

\section{REFERENCES}

Bilinskis, I. and A. Mikelsons (1992). Randomized Signal Processing. Prentice Hall.

Eng, F. and F. Gustafsson (2005). System identification using measurements subject to stochastic time jitter. In: International Federation of Automatic Control (IFAC) World Congress. IFAC. Prague.

Eng, F., F. Gustafsson and F. Gunnarsson (2005). Frequency domain analysis in the case of stochastic sampling times. Submitted to IEEE Transactions on Signal Processing.

Gillberg, J. and F. Gustafsson (2005). Frequencydomain continuous time AR modeling using non-uniformly sampled measurments. In: IEEE Conference on Acoustics, Speech and Signal Processing (ICASSP). IEEE. Philadelphia.

Gillberg, J. and L. Ljung (2005). Frequencydomain identification of continuous-time output error models from sampled data. In: IFAC World Congress.

Ljung, L. (1999). System identification, Theory for the user. second ed.. Prentice Hall. Englewood Cliffs, NJ.

Marvasti, Farokh, Ed. (2001). Nonuniform Sampling: Theory and Practice. Kluwer.

Pintelon, R. and J. Schoukens (2001). System Identification: A Frequency Domain Approach.

Pintelon, R. and J. Schoukens (2005). ML identification of closed-loop systems in a specified frequency band. In: IFAC World Congress.

Schoukens, J., Y. Rolain and R. Pintelon (2005). Analysis of windowing/leakage effects in frequency response function measurements. In: IFAC World Congress.

Souders, T.M., D.R. Flach, C. Hagwood and G.L. Yang (1990). The effects of timing jitter in sampling systems. IEEE Transactions on Instrumentation and Measurements. 